\title{
Existence and concentration of solutions for the nonlinear Kirchhoff type equations with steep well potential
}

Danqing Zhang, Guoqing Chai* and Weiming Liu

"Correspondence:
mathchgq@163.com
School of Mathematics and
Statistics, Hubei Normal University,
Huangshi, 435002, P.R. China

"Correspondence: mathchgq@163.com Statistics, Hubei Normal University, Huangshi, 435002, P.R. China

\begin{abstract}
In this paper, we study the following nonlinear problem of Kirchhoff type:

$$
\left\{\begin{array}{l}
-\left(a+b \int_{\mathbb{R}^{3}}|\nabla u|^{2}\right) \Delta u+\lambda V(x) u=|u|^{p-2} u, \quad \text { in } \mathbb{R}^{3} \\
u \in H^{1}\left(\mathbb{R}^{3}\right)
\end{array}\right.
$$

where the parameter $\lambda>0$ and $4 \leq p<6$, constants $a, b>0$. By variational methods, the results of the existence of nontrivial solutions and the concentration phenomena of the solutions as $\lambda \rightarrow+\infty$ are obtained. It is worth pointing out that, for the case $p \in(4,6)$, the potential $V$ is permitted to be sign-changing.
\end{abstract}

Keywords: Kirchhoff equation; variational methods; concentration

\section{Introduction and main result}

In this paper, we are concerned with the following Kirchhoff type problem:

$$
\left\{\begin{array}{l}
-\left(a+b \int_{\mathbb{R}^{3}}|\nabla u|^{2}\right) \Delta u+\lambda V(x) u=|u|^{p-2} u, \quad \text { in } \mathbb{R}^{3} \\
u \in H^{1}\left(\mathbb{R}^{3}\right),
\end{array}\right.
$$

where $a, b>0$ are constants, $\lambda>0$ is a parameter, $4 \leq p<6$. We assume that $V(x)$ verifies the following hypotheses:

$\left(\mathrm{V}_{1}\right) \quad V \in C\left(\mathbb{R}^{3}, \mathbb{R}\right)$ and $V$ is bounded from below.

$\left(\mathrm{V}_{2}\right)$ There exists $b>0$ such that meas $\left\{x \in \mathbb{R}^{3}: V(x)<b\right\}<\infty$.

$\left(\mathrm{V}_{3}\right)$ The nonempty set $\Omega:=$ int $V^{-1}(0)$ has a smooth boundary and $\bar{\Omega}=V^{-1}(0)$.

In recent years, more and more attention has been devoted to the study of the following Kirchhoff type problems:

$$
\left\{\begin{array}{l}
-\left(a+b \int_{\mathbb{R}^{N}}|\nabla u|^{2}\right) \Delta u+V(x) u=f(x, u), \quad \text { in } \mathbb{R}^{N}, \\
u \in H^{1}\left(\mathbb{R}^{N}\right),
\end{array}\right.
$$

(c) The Author(s) 2017. This article is distributed under the terms of the Creative Commons Attribution 4.0 International License (http://creativecommons.org/licenses/by/4.0/), which permits unrestricted use, distribution, and reproduction in any medium, provided you give appropriate credit to the original author(s) and the source, provide a link to the Creative Commons license, and indicate if changes were made. 
where $V: \mathbb{R}^{N} \rightarrow \mathbb{R}$ and $a, b>0$ are constants. (1.2) is a nonlocal problem as the appearance of the term $\int_{\mathbb{R}^{N}}|\nabla u|^{2}$, which implies that (1.2) is not a pointwise identity. This causes some mathematical difficulties which make the study of (1.2) particularly interesting. If we put $V(x)=0$ and substitute $\mathbb{R}^{N}$ with a bounded domain $\Omega \subset \mathbb{R}^{N}$ in (1.2), then we obtain the following Kirchhoff-Dirichlet problem:

$$
\begin{cases}-\left(a+b \int_{\Omega}|\nabla u|^{2}\right) \Delta u=f(x, u), & x \in \Omega, \\ u=0, & x \in \partial \Omega,\end{cases}
$$

which is associated with the following stationary analogue of the equation:

$$
\rho \frac{\partial^{2} u}{\partial t^{2}}-\left(\frac{P_{0}}{h}+\frac{E}{2 L} \int_{0}^{L}\left|\frac{\partial u}{\partial x}\right|^{2} d x\right) \frac{\partial^{2} u}{\partial x^{2}}=0
$$

presented by Kirchhoff in [16] as an extension of the classical D'Alembert wave equation for free vibrations of elastic strings. For more background, we refer to [4] and the references therein.

Equation (1.2) has been extensively studied in recent years under variant assumptions on $V$ and $f$. In these works, various existence results of the nontrivial solutions to equation (1.2) were established by the variational method. About the existence of infinitely many radial solutions, Jin and Wu in [15] proved the result by applying a fountain theorem for $N=2,3, V(x) \equiv 1$ and $f(x, u)$ is subcritical, superlinear at the origin and 4-superlinear at infinity. When $f(x, u)$ is 4-superlinear at infinity and the potential $V(x)$ satisfies other conditions, Wu in [26] obtained the existence of nontrivial solutions to (1.2) by providing that $(P S)_{c}$ condition holds. In [14], He and Zou proved that (1.2) has a positive ground state solution by using the Nehari manifold. Wang et al. in [24] also proved the multiplicity of positive ground state solutions for (1.2) by the same methods in [14] when $N=3$ and $f(x, u)=\lambda f(u)+|u|^{4} u$. The existence of infinitely many solutions to (1.2) has been derived by a variant version of fountain theorem in [18]. In [19], by using a monotonicity trick and a global compactness lemma, $\mathrm{Li}$ and $\mathrm{Ye}$ obtained the positive ground state for problem (1.2) when $f(x, u)=|u|^{p-2} u$ and $p \in\left(3, \frac{2 N}{N-2}\right)$. Recently, Liu and Guo in [17] extended the above result to $p \in\left(2, \frac{2 N}{N-2}\right)$. For more related results, we refer the readers to $[1-3,5-7$, $9-13,20,21,23,28]$ and the references therein.

In some of the aforementioned references, the potential $V$ is always assumed to be positive or vanishing at infinity. Here, we consider (1.1) with more general potential $V$, especially the potential $V$ can be sign-changing. By a variational method like [27], the existence and concentration of nontrivial solutions of (1.1) are established. We need to overcome some new difficulties, which involves many technical estimates in our paper.

Our main result concerning problem (1.1) is the following.

Theorem 1.1 Suppose that conditions $\left(\mathrm{V}_{1}\right)-\left(\mathrm{V}_{3}\right)$ hold and $4<p<6$. Then there exist positive constants $\Lambda>0$ and $b_{\lambda}^{*}>0$ such that problem (1.1) has at least one nontrivial solution $u_{\lambda} \in H^{1}\left(\mathbb{R}^{3}\right)$ for $\lambda>\Lambda$ in the case of $b>b_{\lambda}^{*}$.

If $V \geq 0$, the following result can be obtained. 
Theorem 1.2 Suppose that conditions $\left(\mathrm{V}_{1}\right)-\left(\mathrm{V}_{3}\right)$ hold. Moreover, $V(x) \geq 0$ and $4 \leq p<6$. Then there exists a constant $\Lambda>0$ such that problem (1.1) has at least one nontrivial solution $u_{\lambda} \in H^{1}\left(\mathbb{R}^{3}\right)$ for $\lambda>\Lambda$.

For the concentration of the solutions of (1.1) as $\lambda \rightarrow+\infty$, we have the following.

Theorem 1.3 Let $u_{\lambda}$ be the solutions obtained in Theorem 1.2, then $u_{\lambda} \rightarrow \bar{u}$ in $H^{1}\left(\mathbb{R}^{3}\right)$ as $\lambda \rightarrow+\infty$, where $\bar{u} \in H_{0}^{1}(\Omega)$ is a nontrivial solution of

$$
\begin{cases}-\left(a+b \int_{\Omega}|\nabla u|^{2}\right) \Delta u=|u|^{p-2} u, & \text { in } \Omega, \\ u=0, & \text { on } \partial \Omega .\end{cases}
$$

The paper is organized as follows. In Section 2, we introduce some notations and the variational framework for (1.1), and then establish compactness conditions. In Section 3, we prove Theorem 1.1 and Theorem 1.2. In the last section, we study the concentration of solutions and prove Theorem 1.3.

\section{Preliminary results}

In this section, we introduce some notations and the variational framework for (1.1) and establish some decomposition of the space to apply the link theorem.

Let $V^{ \pm}(x)=\max \{ \pm V(x), 0\}$. Then $V(x)=V^{+}(x)-V^{-}(x)$. We consider the space

$$
E=\left\{u \in H^{1}\left(\mathbb{R}^{3}\right): \int_{\mathbb{R}^{3}} V^{+} u^{2}<+\infty\right\}
$$

with respect to the inner product and norm defined through

$$
\langle u, v\rangle=\int_{\mathbb{R}^{3}}\left(a \nabla u \nabla v+V^{+} u v\right), \quad\|u\|=\langle u, u\rangle^{\frac{1}{2}} .
$$

For $\lambda>0$, we also consider the following inner product and norm:

$$
\langle u, v\rangle_{\lambda}=\int_{\mathbb{R}^{3}}\left(a \nabla u \nabla v+\lambda V^{+} u v\right), \quad\|u\|_{\lambda}=\langle u, u\rangle_{\lambda}^{\frac{1}{2}} .
$$

We remark that $\|u\| \leq\|u\|_{\lambda}$ for $\lambda \geq 1$. Set $E_{\lambda}=\left(E,\|\cdot\|_{\lambda}\right)$. By $\left(\mathrm{V}_{1}\right),\left(\mathrm{V}_{2}\right)$ and the Poincaré inequality, we can claim that the Hilbert space $E$ is embedded continuously into $H^{1}\left(\mathbb{R}^{3}\right)$. In fact, for any $u \in E$, letting $d=\min \{a, 1\}, V_{b}=\left\{x \in \mathbb{R}^{3}: V(t)<b\right\}, V_{b}^{c}=\left\{x \in \mathbb{R}^{3}: V(x) \geq b\right\}$, we have $\int_{V_{b}} u^{2} \leq c \int_{V_{b}}|\nabla u|^{2}$ for some positive number $c$ by the Poincaré inequality, and therefore

$$
\begin{aligned}
\frac{1}{d}\|u\| & =\frac{1}{d} \int_{\mathbb{R}^{3}}\left(a|\nabla u|^{2}+V^{+} u^{2}\right) \\
& \geq \int_{\mathbb{R}^{3}}\left(|\nabla u|^{2}+V^{+} u^{2}\right) \\
& \geq \int_{\mathbb{R}^{3}}|\nabla u|^{2}+\frac{1}{2} \int_{\mathbb{R}^{3}} V^{+} u^{2} \\
& =\int_{\mathbb{R}^{3}}|\nabla u|^{2}+\frac{1}{2} \int_{V_{b}} V^{+} u^{2}+\frac{1}{2} \int_{V_{b}^{c}} V^{+} u^{2}
\end{aligned}
$$




$$
\begin{aligned}
& \geq \int_{\mathbb{R}^{3}}|\nabla u|^{2}+\frac{1}{2} \int_{V_{b}} V^{+} u^{2}+\frac{b}{2} \int_{V_{b}^{c}} u^{2} \\
& =\frac{1}{2} \int_{\mathbb{R}^{3}}|\nabla u|^{2}+\frac{1}{2} \int_{V_{b}}|\nabla u|^{2}+\frac{1}{2} \int_{V_{b}^{c}}|\nabla u|^{2}+\frac{1}{2} \int_{V_{b}} V^{+} u^{2}+\frac{b}{2} \int_{V_{b}^{c}} u^{2} \\
& \geq \frac{1}{2} \int_{\mathbb{R}^{3}}|\nabla u|^{2}+\frac{1}{2 c} \int_{V_{b}} u^{2}+\frac{b}{2} \int_{V_{b}^{c}} u^{2} \\
& \geq \min \left\{\frac{1}{2}, \frac{1}{2 c}, \frac{b}{2}\right\} \int_{\mathbb{R}^{3}}\left(|\nabla u|^{2}+u^{2}\right) \\
& =\min \left\{\frac{1}{2}, \frac{1}{2 c}, \frac{b}{2}\right\}\|u\|_{H^{1}} .
\end{aligned}
$$

Namely, there exists $\bar{c}>0$ such that $\|u\|_{H^{1}} \leq \bar{c}\|u\|$.

So, for every $t \in[2,6]$, there exists $d_{t}>0$ (independent of $\lambda$ for the case $\lambda \geq 1$ ) such that

$$
|u|_{t} \leq d_{t}\|u\| \leq d_{t}\|u\|_{\lambda} \quad \text { for } u \in E_{\lambda}
$$

Set

$$
F_{\lambda}=\left\{u \in E_{\lambda}: \operatorname{supp} u \subset V^{-1}([0, \infty))\right\}
$$

and $F_{\lambda}^{\perp}$ will be used to denote the orthogonal complement of $F_{\lambda}$ in $E_{\lambda}$. If $V \geq 0$, then $E_{\lambda}=F_{\lambda}$, otherwise $F_{\lambda}^{\perp} \neq 0$. Let $A_{\lambda}=-\Delta+\lambda V$, then $A_{\lambda}$ is formally self-adjoint in $L^{2}\left(\mathbb{R}^{3}\right)$, and the following associated bilinear form:

$$
a_{\lambda}(u, v)=\int_{\mathbb{R}^{3}}(a \nabla u \nabla v+\lambda V(x) u v)
$$

is continuous in $E_{\lambda}$. For fixed $\lambda>0$, study the eigenvalue problem in $F_{\lambda}^{\perp}$ as follows:

$$
-a \Delta u+\lambda V^{+}(x) u=\alpha \lambda V^{-}(x) u
$$

We can get that $u \mapsto \int_{\mathbb{R}^{3}} \lambda V^{-}(x) u^{2} d x$ is weakly continuous since supp $V^{-}$is of finite measure. According to the result in [25], we can obtain a sequence of positive eigenvalues $\alpha_{k}(\lambda)$, which is expressed by

$$
\alpha_{k}(\lambda)=\inf _{k \leq \operatorname{dim} G, G \subset F_{\lambda}^{\perp}} \sup \left\{\|u\|_{\lambda}^{2}: u \in G, \lambda \int_{\mathbb{R}^{3}} V^{-}(x) u^{2}=1\right\}, \quad k=1,2, \ldots
$$

The eigenvalues admit the decompositions: $0<\alpha_{1}(\lambda)<\alpha_{2}(\lambda) \leq \cdots \leq \alpha_{k}(\lambda) \rightarrow+\infty$ as $k \rightarrow$ $+\infty$, and the corresponding eigenfunctions $e_{k}$, which may be chosen so that $\left\langle e_{i}, e_{j}\right\rangle=\delta_{i, j}$ are a basis for $F_{\lambda}^{\perp}$. Let

$$
\widehat{E}_{\lambda}=\operatorname{span}\left\{e_{k}: \alpha_{j}(\lambda) \leq 1\right\} \quad \text { and } \quad E_{\lambda}^{+}=\operatorname{span}\left\{e_{k}: \alpha_{j}(\lambda)>1\right\} .
$$

Then $E_{\lambda}=\widehat{E}_{\lambda} \oplus E_{\lambda}^{+} \oplus F_{\lambda}$ is an orthogonal decomposition with $\operatorname{dim} \widehat{E}_{\lambda}<+\infty$. The bilinear form $a_{\lambda}$ is negative semidefinite on $\widehat{E}_{\lambda}$ and positive definite on $E_{\lambda}^{+} \oplus F_{\lambda}$. If $u, v$ are in different subspaces of the above decomposition of $E_{\lambda}$, then $a_{\lambda}(u, v)=0$. These results will be used later. 
The energy functional associated with (1.1) is

$$
I_{\lambda}(u)=\frac{1}{2} \int_{\mathbb{R}^{3}}\left(a|\nabla u|^{2}+\lambda V(x) u^{2}\right)+\frac{b}{4}\left(\int_{\mathbb{R}^{3}}|\nabla u|^{2}\right)^{2}-\frac{1}{p} \int_{\mathbb{R}^{3}}|u|^{p} .
$$

Let $E$ be a real Banach space and $I: E \rightarrow \mathbb{R}$ be a function of class $C^{1}$. We say that $\left\{u_{n}\right\} \subset E$ is a $(C)_{c}$ sequence if $I\left(u_{n}\right) \rightarrow c$ and $\left(1+\left\|u_{n}\right\|\right) I^{\prime}\left(u_{n}\right) \rightarrow 0$.

Lemma 2.1 Suppose that conditions $\left(\mathrm{V}_{1}\right)-\left(\mathrm{V}_{2}\right)$ hold and $p \in(4,6)$. Then any $(C)_{c}$ sequence of $I_{\lambda}$ is bounded in $E_{\lambda}$ for every $c \in \mathbb{R}$.

Proof Assume that $\left\{u_{n}\right\} \subset E_{\lambda}$ is a $(C)_{c}$ sequence of $I_{\lambda}$. Then

$$
I_{\lambda}\left(u_{n}\right) \rightarrow c,\left(1+\left\|u_{n}\right\|_{\lambda}\right) I^{\prime}\left(u_{n}\right) \rightarrow 0 \quad \text { in } E_{\lambda}^{-1}
$$

Thus, for $n$ large enough, we have

$$
\begin{aligned}
& I_{\lambda}\left(u_{n}\right)-\frac{1}{p}\left\langle I^{\prime}{ }_{\lambda}\left(u_{n}\right), u_{n}\right\rangle \\
& \quad=\left(\frac{1}{2}-\frac{1}{p}\right)\left\|u_{n}\right\|_{\lambda}^{2}-\left(\frac{1}{2}-\frac{1}{p}\right) \int_{\mathbb{R}^{3}} \lambda V^{-}(x) u_{n}^{2}+\left(\frac{1}{4}-\frac{1}{p}\right) b\left(\int_{\mathbb{R}^{3}}\left|\nabla u_{n}\right|^{2}\right)^{2} \\
& \quad \leq c+1 .
\end{aligned}
$$

Combining $\left(\mathrm{V}_{1}\right)$ and (2.5), we deduce that

$$
\begin{aligned}
\left(\frac{1}{2}-\frac{1}{p}\right)\left\|u_{n}\right\|_{\lambda}^{2} & \leq c+1+\left(\frac{1}{2}-\frac{1}{p}\right) \int_{\mathbb{R}^{3}} \lambda V^{-}(x) u_{n}^{2}-\left(\frac{1}{4}-\frac{1}{p}\right) b\left(\int_{\mathbb{R}^{3}}\left|\nabla u_{n}\right|^{2}\right)^{2} \\
& \leq c+1+\left(\frac{1}{2}-\frac{1}{p}\right) \int_{\mathbb{R}^{3}} \lambda V^{-}(x) u_{n}^{2} \\
& \leq c+1+C\left(\frac{1}{2}-\frac{1}{p}\right) \int_{\mathbb{R}^{3}} \lambda u_{n}^{2}
\end{aligned}
$$

where $C>0$ is a constant.

Thus we get

$$
\left\|u_{n}\right\|_{\lambda}^{2} \leq C \int_{\mathbb{R}^{3}} \lambda u_{n}^{2}+(c+1) \frac{2 p}{p-2}
$$

Therefore, it is sufficient to show that $\left\{u_{n}\right\}$ is bounded in $L^{2}\left(\mathbb{R}^{3}\right)$. Assume by contradiction that $\left|u_{n}\right|_{2} \rightarrow+\infty$ as $n \rightarrow \infty$. Let $v_{n}=\frac{u_{n}}{\left|u_{n}\right|_{2}}$, then $\left|v_{n}\right|_{2}=1$. By (2.5) we have

$$
\left\|v_{n}\right\|_{\lambda}^{2}-\lambda \int_{\mathbb{R}^{3}} V^{-}(x) v_{n}^{2}+\frac{p-4}{2(p-2)} b\left(\int_{\mathbb{R}^{3}}\left|\nabla v_{n}\right|^{2}\right)^{2} \int_{\mathbb{R}^{3}} u_{n}^{2} \leq \frac{c+1}{\left(\frac{1}{2}-\frac{1}{p}\right) \int_{\mathbb{R}^{3}} u_{n}^{2}}
$$

and therefore, the sequences $\left\|v_{n}\right\|_{\lambda}$ and $\left(\int_{\mathbb{R}^{3}}\left|\nabla v_{n}\right|^{2}\right)^{2}\left|u_{n}\right|_{2}^{2}$ are both bounded. Up to a subsequence, we have

$$
v_{n} \rightarrow v \quad \text { in } E_{\lambda}, \quad v_{n} \rightarrow v \quad \text { in } L_{\text {loc }}^{s}\left(\mathbb{R}^{3}\right), \quad v_{n} \rightarrow v \quad \text { a.e. in } \mathbb{R}^{3} \text { for } 2 \leq s<6 .
$$


By (2.6) and noting that $\left\|v_{n}\right\|_{\lambda}^{2}-\lambda \int_{\mathbb{R}^{3}} V^{-}(x) v_{n}^{2}=\int_{\mathbb{R}^{3}} a\left|\nabla v_{n}\right|^{2}+\lambda \int_{\mathbb{R}^{3}} V(x) v_{n}{ }^{2}$, we have

$$
\lambda \int_{\mathbb{R}^{3}} V(x) v_{n}^{2} \leq \frac{c+1}{\left(\frac{1}{2}-\frac{1}{p}\right) \int_{\mathbb{R}^{3}} u_{n}^{2}} \rightarrow 0 \quad \text { as } n \rightarrow \infty .
$$

By Fatou's lemma together with (2.6), we see that

$$
\left(\int_{\mathbb{R}^{3}}|\nabla v|^{2}\right)^{2} \leq \lim _{n \rightarrow \infty} \inf \left(\int_{\mathbb{R}^{3}}\left|\nabla v_{n}\right|^{2}\right)^{2} \leq \lim _{n \rightarrow \infty} \inf \frac{C}{\left|u_{n}\right|_{2}^{4}}=0
$$

Hence $v \equiv$ constant. Since $v \in H^{1}\left(\mathbb{R}^{3}\right)$, we infer that $v=0$.

Let $V_{b}=\left\{x \in \mathbb{R}^{3}: V(x)<b\right\}, V_{b}^{c}=\left\{x \in \mathbb{R}^{3}: V(x) \geq b\right\}$. By $\left(\mathrm{V}_{2}\right)$, for any given $\varepsilon>0$, there exists $R_{\varepsilon}>0$ with meas $\left(B_{R_{\varepsilon}}^{c}(0) \cap V_{b}\right)<\varepsilon$, where $B_{R_{\varepsilon}}(0)=\left\{x \in \mathbb{R}^{3}:|x| \leq R_{\varepsilon}\right\}, B_{R_{\varepsilon}}^{c}(0)=$ $\mathbb{R}^{3} \backslash B_{R_{\varepsilon}}(0)$. Therefore, for any fixed $t \in(1,3)$, as $n$ is large enough, we have

$$
\begin{aligned}
\int_{V_{b}} V(x) v_{n}^{2} d x & \leq \int_{B_{R_{\varepsilon}}(0) \cap V_{b}} b v_{n}^{2} d x+\int_{B_{R_{\varepsilon}(0)}^{c}(0) \cap V_{b}} b v_{n}^{2} d x \\
& \leq \varepsilon+b\left|v_{n}\right|_{2 t}^{t} \operatorname{meas}\left(B_{R_{\varepsilon}}^{c}(0) \cap V_{b}\right)^{\frac{t-1}{t}} \\
& \leq c \varepsilon .
\end{aligned}
$$

Therefore, it follows from (2.8) and $\left|v_{n}\right|_{2}^{2}=1$ that

$$
\begin{aligned}
\int_{\mathbb{R}^{3}} V(x) v_{n}^{2} d x & =\int_{V_{b}^{c}} V(x) v_{n}^{2} d x+\int_{V_{b}} V(x) v_{n}^{2} d x \\
& \geq b \int_{V_{b}^{c}} v_{n}^{2} d x+o(1) \\
& \geq b\left(1-\int_{V_{b}} v_{n}^{2} d x\right)+o(1)=b+o(1)>0,
\end{aligned}
$$

which contradicts (2.7). This completes the proof.

Now, we describe the following lemma for the case $p \in[4,6)$ and $V \geq 0$.

Lemma 2.2 Assume that $p \in[4,6), V \geq 0$ and conditions $\left(\mathrm{V}_{1}\right)-\left(\mathrm{V}_{2}\right)$ hold. Then there exists $\Lambda>0$ such that $I_{\lambda}$ satisfies $(C)_{c}$ condition for all $\lambda>\Lambda$ and $c=c_{\lambda}:=\inf _{\gamma \in \Gamma} \max _{t \in[0,1]} I_{\lambda}(\gamma(t))$, which is showed in (3.7) later.

Proof Let $u_{n}$ be a $(C)_{c}$ sequence. By Lemma 2.1, $u_{n}$ is bounded in $E_{\lambda}$ and there exists $C$ such that $\left\|u_{n}\right\|_{\lambda} \leq C$ (for the case $p=4$, that is also true by (2.5) and $V \geq 0$ ).

Hence, without loss of generality, we can say that

$$
u_{n} \rightarrow u \quad \text { in } E_{\lambda}, \quad u_{n} \rightarrow u \quad \text { in } L_{\text {loc }}^{s}\left(\mathbb{R}^{3}\right) \text { for } 2 \leq s<6 \quad \text { and } \quad \int_{\mathbb{R}^{3}}\left|\nabla u_{n}\right|^{2} \rightarrow A^{2}
$$

Firstly, we can claim that $I_{\lambda}^{\prime}(u)=0$ for $4 \leq p<6$.

If $u \equiv 0$, then the claim is finished. 
If $u \not \equiv 0$, then we see

$$
\int_{\mathbb{R}^{3}}|\nabla u|^{2} \leq \lim _{n \rightarrow \infty} \int_{\mathbb{R}^{3}}\left|\nabla u_{n}\right|^{2}=A^{2}
$$

Suppose $\int_{\mathbb{R}^{3}}|\nabla u|^{2}<A^{2}$, since $I_{\lambda}^{\prime}\left(u_{n}\right) \rightarrow 0$ and $\int_{\mathbb{R}^{3}}\left|\nabla u_{n}\right|^{2} \rightarrow A^{2}$, then

$$
\int_{\mathbb{R}^{3}}(a \nabla u \nabla \varphi+\lambda V(x) u \varphi)+b A^{2} \int_{\mathbb{R}^{3}} \nabla u \nabla \varphi-\int_{\mathbb{R}^{3}}|u|^{p-2} u \varphi=0, \quad \forall \varphi \in E_{\lambda} .
$$

Then $I_{\lambda}^{\prime}(u) u<0$. Noting that $I_{\lambda}^{\prime}(t u)(t u)>0$ for small $t>0$ and $\left\langle I_{\lambda}^{\prime}(t u), t u\right\rangle$ is continuous on $t \in[0,1]$. Therefore, there exists $t_{0} \in(0,1)$ such that

$$
\left\langle I_{\lambda}^{\prime}\left(t_{0} u\right), t_{0} u\right\rangle=0
$$

Observing the definition of $c_{\lambda}$ and $I_{\lambda}\left(t_{0} u\right)=\max _{t \in[0,1]} I(t u)$, we have

$$
\begin{aligned}
c_{\lambda} & \leq I_{\lambda}\left(t_{0} u\right)=I_{\lambda}\left(t_{0} u\right)-\frac{1}{4}\left\langle I_{\lambda}^{\prime}\left(t_{0} u\right), t_{0} u\right\rangle \\
& =\frac{t_{0}^{2}}{4} \int_{\mathbb{R}^{3}}\left(a|\nabla u|^{2}+\lambda V(x) u^{2}\right)+\left(\frac{1}{4}-\frac{1}{p}\right) t_{0}^{p} \int_{\mathbb{R}^{3}}|u|^{p} \\
& <\frac{1}{4} \int_{\mathbb{R}^{3}}\left(a|\nabla u|^{2}+\lambda V(x) u^{2}\right)+\left(\frac{1}{4}-\frac{1}{p}\right) \int_{\mathbb{R}^{3}}|u|^{p} \\
& \leq \lim _{n \rightarrow \infty} \inf \left[\frac{1}{4} \int_{\mathbb{R}^{3}}\left(a\left|\nabla u_{n}\right|^{2}+\lambda V(x) u_{n}^{2}\right)+\left(\frac{1}{4}-\frac{1}{p}\right) \int_{\mathbb{R}^{3}}\left|u_{n}\right|^{p}\right] \\
& =\lim _{n \rightarrow \infty} \inf \left[I_{\lambda}\left(u_{n}\right)-\frac{1}{4}\left\langle I_{\lambda}^{\prime}\left(u_{n}\right), u_{n}\right\rangle\right]=c_{\lambda},
\end{aligned}
$$

which is impossible. Then $\int_{\mathbb{R}^{3}}|\nabla u|^{2}=A^{2}=\lim _{n \rightarrow \infty} \int_{\mathbb{R}^{3}}\left|\nabla u_{n}\right|^{2}$, and so $I_{\lambda}^{\prime}(u)=0$ for $4 \leq$ $p<6$. Thus, the claim is got.

Furthermore, from $V \geq 0$ and $p \in[4,6)$, it follows that $a_{\lambda}(u, u)=\|u\|_{\lambda}^{2}$ and

$$
I_{\lambda}(u)=I_{\lambda}(u)-\frac{1}{4}\left\langle I_{\lambda}^{\prime}(u), u\right\rangle=\frac{1}{4}\|u\|_{\lambda}^{2}+\left(\frac{1}{4}-\frac{1}{p}\right)|u|_{p}^{p} \geq 0
$$

Next, we show that $u_{n} \rightarrow u$ in $E_{\lambda}$. Let $v_{n}:=u_{n}-u$.

By $\left(\mathrm{V}_{2}\right)$ and a proof similar to (2.8), we have

$$
\left|v_{n}\right|_{2}^{2}=\int_{\{V(x) \geq b\}} v_{n}^{2} d x+\int_{\{V(x)<b\}} v_{n}^{2} d x \leq \frac{1}{\lambda b}\left\|v_{n}\right\|_{\lambda}^{2}+o(1) .
$$

Then, by Hölder's inequality and Sobolev's embedding theorem, we have

$$
\left|v_{n}\right|_{p}=\left|v_{n}\right|_{2}^{\theta}\left|v_{n}\right|_{6}^{1-\theta} \leq d\left|v_{n}\right|_{2}^{\theta}\left|\nabla v_{n}\right|_{2}^{1-\theta} \leq d(\lambda b)^{-\frac{\theta}{2}}\left\|v_{n}\right\|_{\lambda}+o(1)
$$

as $n \rightarrow+\infty$, where $\theta=\frac{6-p}{2 p}$ and $d$ is a constant independent of $\lambda$.

Applying the Brezis-Lieb lemma, we have

$$
\int_{\mathbb{R}^{3}}\left|\nabla v_{n}\right|^{2}=\int_{\mathbb{R}^{3}}\left|\nabla u_{n}\right|^{2}-\int_{\mathbb{R}^{3}}|\nabla u|^{2}+o(1)
$$


and

$$
\left(\int_{\mathbb{R}^{3}}\left|\nabla v_{n}\right|^{2}\right)^{2}=\left(\int_{\mathbb{R}^{3}}\left|\nabla u_{n}\right|^{2}\right)^{2}-\left(\int_{\mathbb{R}^{3}}|\nabla u|^{2}\right)^{2}+o(1) .
$$

Moreover, we obtain

$$
I_{\lambda}\left(v_{n}\right)=I_{\lambda}\left(u_{n}\right)-I_{\lambda}(u)+o(1) \quad \text { and } \quad I_{\lambda}^{\prime}\left(v_{n}\right) \rightarrow 0 \quad \text { as } n \rightarrow \infty .
$$

Therefore, by (2.9) we have

$$
\begin{aligned}
\frac{1}{4}\left\|v_{n}\right\|_{\lambda}^{2}+\left(\frac{1}{4}-\frac{1}{p}\right)\left|v_{n}\right|_{p}^{p} & =I_{\lambda}\left(v_{n}\right)-\frac{1}{4}\left\langle I_{\lambda}^{\prime}\left(v_{n}\right), v_{n}\right\rangle \\
& =c_{\lambda}-I_{\lambda}(u)+o(1) \leq c_{\lambda}+o(1) .
\end{aligned}
$$

Hence,

$$
\left|v_{n}\right|_{p}^{p-2} \leq\left(\frac{4 p}{p-4}\right)^{\frac{p-2}{p}} c_{\lambda}^{\frac{p-2}{p}}+o(1)<\left(\frac{4 p}{p-3}\right)^{\frac{p-2}{p}} c_{\lambda}^{\frac{p-2}{p}}+o(1) .
$$

If $p=4$, it follows from (2.1) and (2.13) that

$$
\left|v_{n}\right|_{p}^{p-2} \leq d_{p}^{p-2}\left\|v_{n}\right\|_{\lambda}^{p-2} \leq\left(2 d_{p}\right)^{p-2} c_{\lambda}^{\frac{p-2}{2}}+o(1)
$$

where a constant $d_{p}>0$ is independent of $\lambda \geq 1$. Hence, whenever $p>4$ or $p=4$, it follows from (2.14)-(2.15) that

$$
\left|v_{n}\right|_{p}^{p-2} \leq \max \left\{\left(\frac{4 p}{p-3}\right)^{\frac{p-2}{p}} c_{\lambda}^{\frac{p-2}{p}},\left(2 d_{p}\right)^{p-2} c_{\lambda}^{\frac{p-2}{p}}\right\}+o(1) .
$$

Let $b_{\lambda}=\max \left\{\left(\frac{4 p}{p-3}\right)^{\frac{p-2}{p}} c_{\lambda}^{\frac{p-2}{p}},\left(2 d_{p}\right)^{p-2} c_{\lambda}^{\frac{p-2}{p}}\right\}$. Then, in terms of (2.11), we have

$$
\left|v_{n}\right|_{p}^{p}=\left|v_{n}\right|_{p}^{p-2}\left|v_{n}\right|_{p}^{2} \leq b_{\lambda} d^{2}(\lambda b)^{-\theta}\left\|v_{n}\right\|^{2}+o(1)
$$

Since $\left\langle I_{\lambda}^{\prime}\left(v_{n}\right), v_{n}\right\rangle=o(1)$, we have

$$
\begin{aligned}
o(1) & =\left\|v_{n}\right\|_{\lambda}^{2}+b\left(\int_{\mathbb{R}^{3}}\left|\nabla v_{n}\right|^{2}\right)^{2}-\left|v_{n}\right|_{p}^{p} \\
& \geq\left\|v_{n}\right\|_{\lambda}^{2}-\left|v_{n}\right|_{p}^{p} \\
& \geq\left(1-b_{\lambda} d^{2}(\lambda b)^{-\theta}\right)\left\|v_{n}\right\|_{\lambda}^{2}+o(1) .
\end{aligned}
$$

Hence, there exists a positive number $\Lambda$ such that $v_{n} \rightarrow 0$ in $E_{\lambda}$ as $n \rightarrow \infty$ for $\lambda>\Lambda$.

Remark 2.3 About the proof of Lemma 2.2, we can see that formula (2.9) is vital. Since $V$ is sign-changing, for any critical point $u$ of $I_{\lambda}$, it becomes more difficult to induce the result that $I_{\lambda}(u) \geq 0$. Indeed, we have the following corollary. 
Corollary 2.4 Suppose that conditions $\left(\mathrm{V}_{1}\right)-\left(\mathrm{V}_{2}\right)$ hold and $p \in(4,6)$. Let $\left\{u_{n}\right\}$ be a $(C)_{c}$ sequence of $I_{\lambda}$ with level $c=c_{\lambda}>0$, where $c_{\lambda}=\inf _{\gamma \in \Gamma} \max _{u \in Q} I_{\lambda}(\gamma(u)), \Gamma:=\left\{C\left(Q, E_{\lambda}\right):\left.\gamma\right|_{\partial Q}=\right.$ $\left.I_{d}\right\}$, which is mentioned in Proposition 3.1. Then there exists $\Lambda>0$ such that, up to a subsequence, $u_{n} \rightarrow u$ in $E_{\lambda}$. Moreover, the nontrivial critical point of $I_{\lambda}$ satisfies $I_{\lambda}(u) \leq c$ for all $\lambda>\Lambda$.

Proof We adopt an approach similar to the proof of Lemma 2.2. In terms of Lemma 2.1, we know that $\left\{u_{n}\right\}$ is bounded by $c_{\lambda}$ in $E_{\lambda}$. Then $u_{n} \rightarrow u$ in $E_{\lambda}$, and $u$ is a critical point of $I_{\lambda}$. However, since $V$ can be sign-changing and

$$
I_{\lambda}(u)=I_{\lambda}(u)-\frac{1}{4}\left\langle I^{\prime}{ }_{\lambda}(u), u\right\rangle=\frac{1}{4}\|u\|_{\lambda}^{2}-\frac{\lambda}{4} \int_{\mathbb{R}^{3}} V^{-}(x) u^{2}+\left(\frac{1}{4}-\frac{1}{p}\right)|u|_{p}^{p},
$$

we cannot deduce that $I_{\lambda}(u) \geq 0$. Next, we only need to consider the following two cases:

$$
\text { (i) } \quad I_{\lambda}(u)<0 ; \quad \text { (ii) } \quad I_{\lambda}(u) \geq 0 \text {. }
$$

In case (i), obviously, $u$ is a nontrivial solution and the conclusion is obtained.

In case (ii), as in the proof of Lemma 2.2, we can see $u_{n} \rightarrow u$ in $E_{\lambda}$. Let $v_{n}=u_{n}-u$, indeed, by $\left(\mathrm{V}_{2}\right)$ and deduction similar to $(2.8)$, we have

$$
\lambda \int_{\mathbb{R}^{3}} V^{-}(x) v_{n}^{2} d x \rightarrow 0
$$

Therefore, similar to (2.13), we have

$$
\frac{1}{4}\left\|v_{n}\right\|_{\lambda}^{2}+\left(\frac{1}{4}-\frac{1}{p}\right)\left|v_{n}\right|_{p}^{p}+o(1) \leq c_{\lambda}-I_{\lambda}(u)+o(1) \leq c_{\lambda}+o(1) .
$$

So we also have (2.18). Hence $u_{n} \rightarrow u$ in $E_{\lambda}$ and $I_{\lambda}(u)=c>0$ and the proof is finished.

\section{Proof of Theorem 1.1 and Theorem 1.2}

We first give the link theorem [22] under $(C)_{c}$ condition which is useful in the case of $V$ is sign-changing. We will obtain the solutions of (1.1) and give the proofs of Theorem 1.1 and Theorem 1.2.

Proposition 3.1 Let $E=E_{1} \oplus E_{2}$ be a Banach space with $\operatorname{dim} E_{2}<\infty, \Phi \in C^{1}(E, \mathbb{R})$. If there exist $R>\rho>0, \kappa>0$ and $e_{0} \in E_{1}$ such that

$$
\kappa=\inf \Phi\left(E_{1} \cap S_{\rho}\right)>\sup \Phi(\partial Q),
$$

where $S_{\rho}=\{u \in E:\|u\|=\rho\}, Q=\left\{u=v+t e_{0}: v \in E_{2}, t \geq 0,\|u\| \leq R\right\}$. Then $c \geq \kappa$ and $\Phi$ has a $(C)_{c}$ sequence, where $c=\inf _{\gamma \in \Gamma} \max _{u \in Q} I_{\lambda}(\gamma(u)), \Gamma:=\left\{C(Q, E):\left.\gamma\right|_{\partial Q}=I_{d}\right\}$.

Here, we use Proposition 3.1 with $E_{1}=E_{\lambda}^{+} \oplus F_{\lambda}$ and $E_{2}=\widehat{E}_{\lambda}$. For every $j$ fixed, by Lemma 2.1 in [8], we have $\alpha_{j}(\lambda) \rightarrow 0$ as $\lambda \rightarrow \infty$. Hence, for $\lambda>\Lambda_{0}, \widehat{E}_{\lambda}$ is the finite dimensional space and there is $\Lambda_{0}>0$ such that $\widehat{E}_{\lambda} \neq \emptyset$. All of this indicates that there exists $\widehat{C}_{\lambda}>0$ with

$$
|u|^{p} \geq \widehat{C}_{\lambda}\|u\| \quad \text { for } u \in \widehat{E}_{\lambda},
$$


where $\widehat{C}_{\lambda}$ is a constant dependent on $\lambda$. Now we will verify that the functional $I_{\lambda}$ satisfies the linking structure.

Lemma 3.2 For each $\lambda>\Lambda_{0}$, there exist $\rho_{\lambda}>0$ and $\kappa_{\lambda}>0$ such that $I_{\lambda}(u) \geq \kappa_{\lambda}$ for all $u \in E_{\lambda}^{+} \oplus F_{\lambda}$ with $\|u\|_{\lambda}=\rho_{\lambda}$. Furthermore, as $V \geq 0$, we can choose the constants $\rho$ and $\kappa$ independent of $\lambda$ for the case $\lambda \geq 1$.

Proof By the definition of $E_{\lambda}^{+}$, there exists $\delta_{\lambda}>0$ such that

$$
a_{\lambda}(u, u) \geq \delta_{\lambda}\|u\|_{\lambda}^{2} \quad \text { for } u \in E_{\lambda}^{+}
$$

and

$$
a_{\lambda}(u, u)=\|u\|_{\lambda}^{2} \text { for } u \in F_{\lambda} .
$$

Therefore, for $u=v+w \in E_{\lambda}^{+} \oplus F_{\lambda}$, since $\langle v, w\rangle_{\lambda}=0$ and $a_{\lambda}(v, w)=0$ as mentioned before, we have

$$
\begin{aligned}
I_{\lambda}(u) & =\frac{1}{2} a_{\lambda}(v, v)+\frac{1}{2} a_{\lambda}(w, w)+\frac{b}{4}\left(\int_{\mathbb{R}^{3}}|\nabla u|^{2}\right)^{2}-\frac{1}{p}|u|_{p}^{p} \\
& \geq \frac{1}{2} \min \left\{\delta_{\lambda}, 1\right\}\|u\|_{\lambda}^{2}-\bar{c}\|u\|_{\lambda}^{p},
\end{aligned}
$$

where the constant $\bar{c}$ is independent of $\lambda \geq 1$.

By (2.1), we can choose $\rho_{\lambda}>0$ and small $\kappa_{\lambda}$ such that the first half of the lemma holds. If $V \geq 0$, note that $a_{\lambda}(u, u)=\|u\|_{\lambda}^{2}$, thus we finally have the conclusion.

Now, we choose $e_{0} \in C_{0}^{\infty}(\Omega)$ which will be used in the following lemma, by $\left(\mathrm{V}_{3}\right)$, we have $e_{0} \in F_{\lambda}$.

Lemma 3.3 Suppose that assumptions in Theorem 1.1 hold. For each $\lambda>\Lambda_{0}$, there exist $b^{*}(\lambda)>0$ and $R_{\lambda}>\rho_{\lambda}$ such that for $b<b^{*}(\lambda)$

$$
\sup _{u \in \partial Q} I_{\lambda}(u)<\kappa_{\lambda}
$$

where $Q=\left\{u=v+t e_{0}: v \in \widehat{E}_{\lambda}, t \geq 0,\|u\| \leq R_{\lambda}\right\}, \kappa_{\lambda}$ and $\rho_{\lambda}$ mentioned in Lemma 3.2.

Proof (i) For $u=v+w \in \widehat{E}_{\lambda} \oplus \mathbb{R} e_{0}$, since $a_{\lambda}(v, w)=0$ as before, we have

$$
a_{\lambda}(u, u)=a_{\lambda}(v, v)+a_{\lambda}(w, w) .
$$

We show that $a_{\lambda}(v, v) \leq 0$.

In fact, assume that $\hat{E}_{\lambda}=L\left(e_{1}, e_{2}, \ldots, e_{m}\right)$, and $e_{j}$ is an eigenfunction corresponding to eigenvalue $\alpha_{j}(\lambda)$ with $0<\alpha_{j}(\lambda) \leq 1, j=1,2, \ldots$. It follows from (2.2) that

$$
\left\langle e_{j}, \phi\right\rangle_{\lambda}=\alpha_{j}(\lambda) \lambda \int_{\mathbb{R}^{3}} V^{-}(x) e_{j} \phi, \quad \forall \phi \in E_{\lambda} .
$$


Thus, noting that $0<\alpha_{j}(\lambda) \leq 1$, we have

$$
\left\langle e_{j}, e_{j}\right\rangle_{\lambda} \leq \lambda \int_{\mathbb{R}^{3}} V^{-}(x) e_{j}^{2},
$$

and therefore $a_{\lambda}\left(e_{j}, e_{j}\right) \leq 0$. Similarly, by (3.2) we also have

$$
0=\left\langle e_{j}, e_{i}\right\rangle_{\lambda}=\alpha_{j}(\alpha) \lambda \int_{\mathbb{R}^{3}} V^{-}(x) e_{j} e_{i}, \quad i \neq j .
$$

Now, noting that $\left\{e_{j}\right\}$ is a base of $\hat{E}_{\lambda}$, we can prove that $a_{\lambda}(v, v) \leq 0$. Hence, we have

$$
a_{\lambda}(u, u) \leq a_{\lambda}(w, w)=a|\nabla w|_{2}^{2} \leq\|u\|^{2}
$$

In view of the equivalence of all the norms on a finite dimensional space, we obtain

$$
I_{\lambda}(u) \leq \frac{1}{2}\|u\|^{2}+\frac{b}{4}|\nabla u|_{2}^{4}-\frac{1}{p}|u|^{p} \rightarrow-\infty
$$

for $u \in \widehat{E}_{\lambda} \oplus \mathbb{R} e_{0}$ with $\|u\|_{\lambda} \rightarrow+\infty$. As a result, there exists $R_{\lambda}>0$ such that $I_{\lambda}(u) \leq \kappa_{\lambda}$ for $u \in \widehat{E}_{\lambda} \oplus \mathbb{R} e_{0}$ satisfying $\|u\|_{\lambda}=R_{\lambda}$.

(ii) For $u \in \widehat{E}_{\lambda}$ with $\|u\|_{\lambda} \leq R_{\lambda}$, we have

$$
I_{\lambda}(u) \leq \frac{b}{4}\left(\int_{\mathbb{R}^{3}}|\nabla u|^{2}\right)^{2} \leq \frac{b}{4}\|u\|_{\lambda}^{4} \leq \frac{b}{4} R_{\lambda}^{4} .
$$

Therefore, taking $b^{*}(\lambda)=\frac{4 \kappa_{\lambda}}{R_{\lambda}^{4}}$, we obtain the conclusion.

Proof of Theorem 1.1 By Lemmas 3.2-3.3 and applying Proposition 3.1, it follows that for any $\lambda>\Lambda_{0}$ and $0<b<b_{\lambda}^{*}, I_{\lambda}$ possesses a $(C)_{c}$ sequence $\left\{u_{n}\right\}$ with $c=c_{\lambda}$. Now, by Lemma 2.1 and Corollary 2.4, we can obtain the conclusion of Theorem 1.1.

Proof of Theorem 1.2 For the case $V \geq 0$, we can easily prove that the functional $I$ satisfies the conditions of mountain-pass theorem, and therefore, the existence of nontrivial solutions can be obtained.

Since $V(x) \geq 0$, we have

$$
\begin{aligned}
I_{\lambda}(u) & =\frac{1}{2}\|u\|_{\lambda}^{2}+\frac{b}{4}\left(\int_{\mathbb{R}^{3}}|\nabla u|^{2}\right)^{2}-\frac{1}{p} \int_{\mathbb{R}^{3}}|u|^{p} \\
& \geq \frac{1}{2}\|u\|_{\lambda}^{2}-\frac{1}{p} \int_{\mathbb{R}^{3}}\|u\|_{\lambda}^{p} .
\end{aligned}
$$

Hence there exist two positive numbers $\alpha, \rho$ such that $I_{\lambda}(u) \geq \alpha$ for $\|u\|_{\lambda}=\rho$ small enough.

Let $e_{0} \in C_{0}^{\infty}(\Omega)$, then

$$
\begin{aligned}
I_{\lambda}\left(t e_{0}\right) & =\frac{t^{2}}{2} \int_{\mathbb{R}^{3}}\left(a\left|\nabla e_{0}\right|^{2}+\lambda V(x) e_{0}^{2}\right)+\frac{b}{4} t^{4}\left(\int_{\mathbb{R}^{3}}\left|\nabla e_{0}\right|^{2}\right)^{2}-\frac{t^{p}}{4} \int_{\mathbb{R}^{3}}\left|e_{0}\right|^{p} d x \\
& \rightarrow-\infty
\end{aligned}
$$


as $t \rightarrow \infty$. Then there exists $t_{0}>0$ large such that

$$
I_{\lambda}\left(t_{0} e_{0}\right)<0 \quad \text { and } \quad\left\|t_{0} e_{0}\right\|_{\lambda}>\rho .
$$

By the mountain-pass theorem, there exists a $(C)_{c}$ sequence $\left\{u_{n}\right\} \subset E_{\lambda}$ such that

$$
I_{\lambda}\left(u_{n}\right) \rightarrow c_{\lambda}, I_{\lambda}^{\prime}\left(u_{n}\right) \rightarrow 0 \quad \text { in } E_{\lambda}^{-1},
$$

where

$$
0<c_{\lambda}=: \inf _{\gamma \in \Gamma} \max _{t \in[0,1]} \mathrm{I}_{\lambda}(\gamma(t)) \leq \sup _{t \geq 0} I_{\lambda}\left(t e_{0}\right) \leq C_{0},
$$

$\Gamma=\left\{\gamma \in C\left([0,1], E_{\lambda}\right): \gamma(0)=0,\|\gamma(1)\|_{\lambda}>\rho, I_{\lambda}(\gamma(1))<0\right\}$.

By Lemma 2.2, for $\lambda$ large enough, we can get a nontrivial critical point $u$ for $I_{\lambda}$ with $I_{\lambda}\left(u_{\lambda}\right) \in\left[c_{\lambda}, C_{0}\right]$.

\section{Concentration for solutions}

Now, using the same notation as before, we are ready to investigate the concentration for solutions and give the proof of Theorem 1.3.

Proof For any sequence $\lambda_{n} \rightarrow+\infty$, let $u_{n}:=u_{\lambda_{n}}$ be the critical points of $I_{\lambda_{n}}$ obtained in Theorem 1.2.

It follows from (3.7) and

$$
I_{\lambda_{n}}\left(u_{n}\right)=I_{\lambda_{n}}\left(u_{n}\right)-\frac{1}{4}\left\langle I_{\lambda_{n}}^{\prime}\left(u_{n}\right), u_{n}\right\rangle=\frac{1}{4}\left\|u_{n}\right\|_{\lambda_{n}}^{2}+\left(\frac{1}{4}-\frac{1}{p}\right)\left|u_{n}\right|_{p}^{p}
$$

that

$$
\sup _{n \geq 1}\left\|u_{n}\right\|_{\lambda_{n}}^{2} \leq 4 C_{0}
$$

where the constant $C_{0}$ is independent of $\lambda_{n}$.

Therefore, we may assume that $u_{n} \rightarrow \bar{u}$ in $E$ and $u_{n} \rightarrow \bar{u}$ in $L_{\mathrm{loc}}^{s}\left(\mathbb{R}^{3}\right)$ for $2 \leq s<6$. By Fatou's lemma, we deduce

$$
\int_{\mathbb{R}^{3}} V(x) \bar{u}^{2} \leq \lim _{n \rightarrow \infty} \inf \int_{\mathbb{R}^{3}} V(x) u_{n}^{2} \leq \lim _{n \rightarrow \infty} \inf \frac{\left\|u_{n}\right\|_{\lambda_{n}}^{2}}{\lambda_{n}}=0 .
$$

Therefore $\bar{u}=0$ a.e in $\mathbb{R}^{3} \backslash V^{-1}(0)$, and so $\bar{u} \in H_{0}^{1}(\Omega)$ by $\left(\mathrm{V}_{3}\right)$.

Now, for any $\varphi \in C_{0}^{\infty}(\Omega)$, since $\left\langle I^{\prime}{ }_{\lambda_{n}}\left(u_{n}\right), \varphi\right\rangle=0$, we obtain

$$
\int_{\Omega} a \nabla \bar{u} \nabla \varphi+b \int_{\Omega}|\nabla \bar{u}|^{2} \int_{\Omega} \nabla \bar{u} \nabla \varphi=\int_{\Omega}|\bar{u}|^{p-2} \bar{u} \varphi .
$$

By the density of $C_{0}^{\infty}(\Omega)$ in $H_{0}^{1}(\Omega), \bar{u}$ is a weak solution of (1.4).

Next, we need to prove that $u_{n} \rightarrow \bar{u}$ in $L^{s}\left(\mathbb{R}^{3}\right)$ for $s \in(2,6)$. If not, from the vanishing lemma, it follows that there exist two positive constants $\delta, \rho$ and $x_{n} \in \mathbb{R}^{3}$ such that

$$
\int_{B_{\rho}\left(x_{n}\right)}\left(u_{n}-\bar{u}\right)^{2} \geq \delta
$$


Moreover, $\left|x_{n}\right| \rightarrow \infty$. Therefore meas $\left(B_{\rho}\left(x_{n}\right) \cap\left\{x \in \mathbb{R}^{3}: V(x)<b\right\}\right) \rightarrow 0$. By Hölder's inequality and an argument similar to that used in the proof of (2.8), we have

$$
\int_{B_{\rho}\left(x_{n}\right) \cap\{V(x)<b\}}\left(u_{n}-\bar{u}\right)^{2} d x \rightarrow 0 .
$$

Consequently,

$$
\begin{aligned}
\left\|u_{n}\right\|_{\lambda_{n}}^{2} & \geq \lambda_{n} b \int_{B_{\rho}\left(x_{n}\right) \cap\{V(x) \geq b\}} u_{n}^{2} d x=\lambda_{n} b \int_{B_{\rho}\left(x_{n}\right) \cap\{V(x) \geq b\}}\left(u_{n}-\bar{u}\right)^{2} d x \\
& =\lambda_{n} b\left(\int_{B_{\rho}\left(x_{n}\right)}\left(u_{n}-\bar{u}\right)^{2} d x+o(1)\right) \rightarrow+\infty,
\end{aligned}
$$

which contradicts (4.1).

Last, we only need to prove that $u_{n} \rightarrow \bar{u}$ in $E$. Since $\left\langle I^{\prime}{ }_{\lambda_{n}}\left(u_{n}\right), u_{n}\right\rangle=\left\langle I^{\prime}{ }_{\lambda_{n}}\left(u_{n}\right), \bar{u}\right\rangle=0$, we have

$$
\left\|u_{n}\right\|_{\lambda_{n}}^{2}+b\left(\int_{\mathbb{R}^{3}}\left|\nabla u_{n}\right|^{2}\right)^{2}=\int_{\mathbb{R}^{3}}\left|u_{n}\right|^{p}
$$

and

$$
\left\langle u_{n}, \bar{u}\right\rangle_{\lambda_{n}}+b \int_{\mathbb{R}^{3}}\left|\nabla u_{n}\right|^{2} \int_{\mathbb{R}^{3}} \nabla u_{n} \nabla \bar{u}=\int_{\mathbb{R}^{3}}\left|u_{n}\right|^{p-2} u_{n} \bar{u} .
$$

We can prove that

$$
\left(\int_{\mathbb{R}^{3}}\left|\nabla u_{n}\right|^{2}\right)^{2}-\int_{\mathbb{R}^{3}}\left|\nabla u_{n}\right|^{2} \int_{\mathbb{R}^{3}} \nabla u_{n} \nabla \bar{u} \rightarrow 0 .
$$

Combining (4.2), (4.3) and (4.4), we obtain

$$
\lim _{n \rightarrow \infty}\left\|u_{n}\right\|_{\lambda_{n}}^{2}=\lim _{n \rightarrow \infty}\left\langle u_{n}, \bar{u}\right\rangle_{\lambda_{n}}=\lim _{n \rightarrow \infty}\left\langle u_{n}, \bar{u}\right\rangle=\|\bar{u}\|^{2}
$$

Thanks to the weak lower semi-continuity, we have

$$
\|\bar{u}\|^{2} \leq \lim _{n \rightarrow \infty} \inf \left\|u_{n}\right\|^{2} \leq \lim _{n \rightarrow \infty} \inf \left\|u_{n}\right\|_{\lambda_{n}}^{2}
$$

so, up to a subsequence, $\left\|u_{n}\right\| \rightarrow\|\bar{u}\|$. Thus, it follows from $u_{n} \rightarrow \bar{u}$ in a Hilbert space $E$ that $u_{n} \rightarrow \bar{u}$ in $E$.

Since $u_{n} \neq 0$, by $(4.2)$ we have

$$
\left\|u_{n}\right\|^{2} \leq\left\|u_{n}\right\|_{\lambda_{n}}^{2} \leq\left|u_{n}\right|_{p}^{p} \leq C\left\|u_{n}\right\|^{p}
$$

which implies that $\bar{u} \neq 0$. Then we can obtain the conclusion. 


\section{Conclusion}

In this paper, by using the variational methods, the existence of nontrivial solutions and the concentration phenomena of the solutions to equation (1.1) were established. We consider (1.1) with more general potential $V$, especially the potential $V$ can be sign-changing. (1.1) is a nonlocal problem as the appearance of the term $\int_{\mathbb{R}^{N}}|\nabla u|^{2}$, so we need to overcome some new difficulties, which involves many technical estimates in our paper.

\section{Funding}

The second author and the third author are supported by the National Natural Science Foundation of China (Grant No. 11601139).

\section{Competing interests}

The authors declare that they have no competing interests.

\section{Authors' contributions}

All authors have made the same contribution and finalized the current version of this manuscript. All authors read and approved the final manuscript.

\section{Publisher's Note}

Springer Nature remains neutral with regard to jurisdictional claims in published maps and institutional affiliations.

Received: 13 July 2017 Accepted: 15 September 2017 Published online: 29 September 2017

\section{References}

1. Alves, CO, Corrêa, F: On existence of solutions for a class of problem involving a nonlinear operator. Commun. Appl. Nonlinear Anal. 8, 43-56 (2001)

2. Alves, CO, Figueiredo, GM: On multiplicity and concentration of positive solutions for a class of quasilinear problems with critical exponential growth in $\mathbb{R}^{N}$. J. Differ. Equ. 246, 1288-1311 (2009)

3. Arosio, A, Panizzi, S: On the well-posedness of the Kirchhoff string. Trans. Am. Math. Soc. 348, 305-330 (1996)

4. Berestycki, H, Lions, PL: Nonlinear scalar field equations I. Arch. Ration. Mech. Anal. 82, 313-345 (1983)

5. D'Aprile, T, Mugnai, D: Non-existence results for the coupled Klein-Gordon-Maxwell equations. Adv. Nonlinear Stud. 4, 307-322 (2004)

6. Deng, Y, Peng, S, Shuai, W: Existence and asymptotic behavior of nodal solutions for the Kirchhoff-type problems in $\mathbb{R}^{3}$. J. Funct. Anal. 269, 3500-3527 (2015)

7. D'ancona, P, Spagnolo, S: Global solvability for the degenerate Kirchhoff equation with real analytic data. Invent. Math. 108, 247-262 (1992)

8. Ding, Y, Szulkin, A: Bound states for semilinear Schrödinger equations with sign-changing potential. Calc. Var. Partial Differ. Equ. 29, 397-419 (2007)

9. Figueiredo, GM, Ikoma, N, Júnior, JRS: Existence and concentration result for the Kirchhoff equations with general nonlinearities. Arch. Ration. Mech. Anal. 213, 931-979 (2014)

10. Furtado, MF, Maia, LA, Medeiros, ES: Positive and nodal solutions for a nonlinear Schrödinger equation with indefinite potential. Adv. Nonlinear Stud. 8, 353 (2008)

11. Guo, Z: Ground states for Kirchhoff equations without compact condition. J. Differ. Equ. 259, 2884-2902 (2015)

12. He, Y: Concentrating bounded states for a class of singularly perturbed Kirchhoff type equations with a general nonlinearity. J. Differ. Equ. 261, 6178-6220 (2016)

13. He, Y, Li, G: Standing waves for a class of Kirchhoff type problems in $\mathbb{R}^{3}$ involving critical Sobolev exponents. Calc. Var. Partial Differ. Equ. 54, 3067-3106 (2015)

14. He, X, Zou, W: Existence and concentration behavior of positive solutions for a Kirchhoff equation in $\mathbb{R}^{3}$. J. Differ. Equ. $252,1813-1834(2012)$

15. Jin, J, Wu, X: Infinitely many radial solutions for Kirchhoff-type problems in $\mathbb{R}^{N}$. J. Math. Anal. Appl. 369, 564-574 (2010)

16. Kirchhoff, G, Hensel, K: Vorlesungen über mathematische physik: bd. Vorlesungen über mechanik. Teubner, Leipzig (1883)

17. Liu, Z, Guo, S: Existence of positive ground state solutions for Kirchhoff type problems. Nonlinear Anal. 120, 1-13 (2015)

18. Liu, W, He, X: Multiplicity of high energy solutions for superlinear Kirchhoff equations. J. Appl. Math. Comput. 39, 473-487 (2012)

19. Li, G, Ye, H: Existence of positive ground state solutions for the nonlinear Kirchhoff type equations in $\mathbb{R}^{3}$. J. Differ. Equ. 257, 566-600 (2014)

20. Pokhozhaev, SI: On a class of quasilinear hyperbolic equations. Mat. Sb. 138, 152-166 (1975)

21. Pucci, P, Saldi, S: Critical stationary Kirchhoff equations in $\mathbb{R}^{N}$ involving nonlocal operators. Rev. Mat. Iberoam. 32, 1-22 (2016)

22. Li, G, Wang, C: The existence of a nontrivial solution to a nonlinear elliptic problem of liking type without the Ambrosetti-Rabinowitz condition. Ann. Acad. Sci. Fenn., Math. 36, 461-480 (2011)

23. Shuai, W: Sign-changing solutions for a class of Kirchhoff-type problem in bounded domains. J. Differ. Equ. 259 1256-1274 (2015) 
24. Wang, J, Tian, L, Xu, J, Zhao, F: Multiplicity and concentration of positive solutions for a Kirchhoff type problem with critical growth. J. Differ. Equ. 253, 2314-2351 (2012)

25. Willem, M: Analyse Harmonique Réelle. Hermann, Paris (1995)

26. $W u, X$ : Existence of nontrivial solutions and high energy solutions for Schrödinger-Kirchhoff-type equations in $\mathbb{R}^{N}$ Nonlinear Anal., Real World Appl. 12, 1278-1287 (2011)

27. Zhao, L, Liu, H, Zhao, F: Existence and concentration of solutions for the Schrödinger-Poisson equations with steep well potential. J. Differ. Equ. 255, 1-23 (2013)

28. Zhao, L, Zhao, F: On the existence of solutions for the Schrödinger-Poisson equations. J. Math. Anal. Appl. 346, 155-169 (2008)

Submit your manuscript to a SpringerOpen ${ }^{\circ}$ journal and benefit from:

- Convenient online submission

- Rigorous peer review

- Open access: articles freely available online

- High visibility within the field

- Retaining the copyright to your article

Submit your next manuscript at $\gg$ springeropen.com 